\title{
DE LA TEORÍA A LA PRÁCTICA LEXICOGRÁFICA: EL «NUEVO DICCIONARIO DE LA LENGUA CASTELLANA» DE VICENTE SALVÁ
}

\author{
Dolores Azorín Fernández- Rosario Baquero Mesa \\ (Universidad de Alicante)
}

\begin{abstract}
RESUMEN
The principal aim of this article is to evaluate the original contribution of Vicente Salvá towards monolingual lexicography of Spanish language in nineteenth century. In order to achieve this objective, we start from the context in which this author is inscribed, contrasting his labour with that developed by the other lexicographs of his generation and, very especially, with the work of the Spanish Royal Academy whose Dictionary (DRAE, 1843) Salvá tried to improve with remarkable success, in our opinion.
\end{abstract}

\section{Los diccionarios monolingües del español en el siglo XIX.}

Vicente Salvá (1786-1849) ${ }^{1}$ es fundamentalmente conocido en el campo de la filología española por ser el autor de una de las gramáticas más difundidas del siglo XIX. En efecto, su Gramática castellana ${ }^{2}$ se puede considerar, junto a las de A. Bello y la Academia, como uno de los textos más representativos de este periodo. Sin embargo, su faceta de lexicógrafo, parangonable en importancia e

1 Para la biografía de nuestro autor, véase: Carola Reig Salvá, Vicente Salvá. Un valenciano de prestigio internacional,Valencia, Instituto Alfonso el Magnánimo, 1972.

2 V. Salvá, Gramática de la lengua castellana, 2 vols., estudio y edición crítica de M. Lliteras, Madrid, Arco-libros, 1988. 
interés a la de gramático, ha permanecido injustamente relegada hasta nuestros días ${ }^{3}$.

La contribución de Salvá a la lexicografía española se inserta en una etapa especialmente importante de su constitución y desarrollo. Si hasta bien entrado el siglo XIX, la Academia Española brillaba casi en solitario merced a su denodada labor en este terreno ${ }^{4}$, a partir del segundo cuarto de esta misma centuria, la situación cambia de manera radical con la aparición en el mercado de un extenso conjunto de diccionarios que se ofrecen, en principio, como superadores del caudal léxico del DRAE (Diccionario de la Real Academia Española), si bien todos ellos, sin excepción, lo utilizan como fuente principal o, en algunos casos, son plagios descarados del texto académico.

Esta nueva corriente lexicográfica se inicia en 1825 con el Diccionario castellano de Manuel Núñez de Taboada ${ }^{5}$, al que pronto se sumarán las obras de Pla y Torres ${ }^{6}$, Peñalver ${ }^{7}$, Labernia ${ }^{8}$, Domínguez ${ }^{9}$, Castro ${ }^{10}$, Gaspar y Roig ${ }^{11}$,

3 En los últimos años, no obstante, la labor lexicográfica de Salvá ha sido puesta de relieve en diversas monografías dedicadas a la historia de nuestros diccionarios. Sobre la contribución de Salvá al panorama lexicográfico de su época se puede consultar: Manuel Seco, «El nacimiento de la lexicografía española no académica», Estudios de lexicografía española, Madrid, Paraninfo, 1987, pp.129-151.También los trabajos de Rosario Baquero, «Notas en contribución a la historia de la lexicografía española monolingüe del siglo XIX», Actas de IV Congreso Internacional de EURALEX, Barcelona, Biblograf, 1992, pp.455-461 y Dolores Azorín, «La lexicografía española en el siglo XIX. Desarrollos y tendencias», Actas de I Congreso de Lingǘstica General, Valencia, 1994 (en prensa). Del tratamiento otorgado a los americanismos en el Nuevo diccionario de Salvá se han ocupado: Manuel Alvar Ezquerra, «La recepción de americanismos en los diccionarios generales de la lengua», Actas de I Congreso Internacional sobre el Español de América, San Juan de Puerto Rico, 1987, pp. 209-218; y Dolores Azorín y Rosario Baquero, «Los americanismos en el Nuevo diccionario de la lengua castellana de Vicente Salvá», Actas del II Congreso Internacional de Historia de la Lengua Española, T.I, Madrid, Arco-Libros, 1992, pp. 963-970.

4 En efecto, el monopolio de la Real Academia en el campo de la lexicografía española monolingüe, iniciado en 1726 con la aparición del primer tomo del Diccionario de Autoridades, sólo se verá interrumpido con la publicación del Diccionario castellano con las vaces de ciencias y artes, del jesuíta Esteban de Terreros y Pando (Madrid, 1786-1793). Existe edición facsímil reciente de este diccionario, a cargo de Manuel Alvar Ezquerra, Madrid, Arco-Libros, 1987, 4 vols. Véase especialmente la «Presentación» que aparece al frente de la mencionada edición, t. I, pp. 516; recogida también en M. Alvar Ezquerra, La lexicografía descriptiva, Barcelona, Biblograf, 1993, pp.249-259.

s Manuel Núñez de Taboada, Diccionario castellano, París, Seguin, 1925.

- Cristóbal Pla y Torres, Diccionario de la lengua castellana por la Academia Española, París, Librería de Cormon y Blanc, 1826.

7 Juan Peñalver, Panléxico. Diccionario universal de la lengua española, Madrid, Imprenta de Ignacio Boix, 1842.

- Pedro Labernia, Diccionario de la lengua castellana con las correspondencias catalana y latina, Barcelona, Imprenta D.J.M. de Grau, 1844-1846.

9 Ramón Joaquín Domínguez, Diccionario nacional o gran diccionario clásico de la lengua española, Madrid, Bernat, 1846-1847.

10 Adolfo de Castro, Gran diccionario de la lengua española, Madrid, Oficinas y Establecimiento Tipográfico del Semanario Pintoresco y de la Ilustración, 1852.

11 Diccionario enciclopédico de la lengua española, revisado por Eduardo Chao, Madrid, Imprenta y Librería de Gaspar y Roig, 1853-1855. 
Sociedad Literaria ${ }^{12}$ y otros repertorios de menor relieve que irrumpen en el mercado, dando lugar a una situación hasta entonces inusitada en la exigua historia de la lexicografia española monolingüe.

Es cierto que nuestra tradición lexicográfica ${ }^{13}$ se encuentra jalonada de importantes hitos que, en su momento, contribuyeron de manera decisiva a la creación del modelo de diccionario moderno: el ejemplo emblemático de Nebrija ${ }^{14}$, inspirador de una vasta progenie de diccionarios bilingües ${ }^{15}$, es la mejor prueba de ello. Sin embargo, no les cupo igual fortuna a los diccionarios monolinguies del español: ni el Tesoro de Covarrubias halló continuadores dignos de su talla ${ }^{16}$, ni el Diccionario de Autoridades (1726-1739) lograría tampoco consolidarse como modelo ${ }^{17}$ para las futuras actuaciones de la Academia, a pesar de haber sido, como afirma M. Seco ${ }^{18}$, «probablemente el mejor de Europa en todo el siglo XVIII». La misma suerte correría el Diccionario de Terreros y Pando (1786-1793 $)^{19}$, único exponente de la lexicografía extra-académica posterior al Diccionario de Autoridades.

12 Nuevo diccionario de la lengua castellana, por una Sociedad Literaria, París, Rosa y Bouret, 1853.

13 Cfr. M. Alvar Ezquerra, «Tradición en los diccionarios del español», Revista de la Sociedad Española de Lingüística, 22, 1, 1992, pp. 1-23.

14 A Elio Antonio de Nebrija le cabe el honor de haber dado principio a la lexicografía modema en Europa. Su Lexicon hoc est Dictionarium ex sermone latino in hispaniensem o Diccionario latino-español, Salamanca, 1492 y su Dictionarium ex hispaniensi in latinum sermone o Vocabulario español-latín, Salamanca, ¿1495?, representan la ruptura con la tradición medieval precedente y el inicio de una nueva manera de concebir el diccionario.

15 La influencia de los diccionarios de Nebrija en la lexicografía modema europea, sobre todo en sus vertientes bilingüe y multilingüe, ha sido puesta de manifiesto, en reiteradas ocasiones, por la crítica especializada. Véanse, a este propósito, los siguientes trabajos: Annamaria Gallina, Contributi alla storia della lessicografia italo-spagnola dei secoli XVI e XVII, Florencia, 1959. M. Alvar Ezquerra, «Antiguos diccionarios plurilingües del español», Actas del I Coloquio Internacional de Traductologia, Valencia, Universidad de Valencia, 1991, pp. 7-14. Gloria Guerrero Ramos, «La lexicografía bilingüe de Nebrija a Oudin», Actas del IV Congreso Internacional de EURALEX, Barcelona, Biblograf, 1992, pp. 463-471.

16 Sebastián de Covarrubias, Tesoro de la lengua castellana o española, Madrid, 1611. Existe edición preparada por Martín de Riquer, Barcelona, 1943 (reimpreso nuevamente, Barcelona, Alta Fulla, 1987), con las adiciones de Benito Remigio Noydens a la edición de 1673-74. A pesar de ser el primer diccionario extenso del español y el primero en Europa de una lengua vulgar, el Tesoro no conseguiría abrir el camino para la instauración de una sólida corriente monolingüe. Sobre la labor de Covarrubias y la repercusión de su obra, véanse: Manuel Seco, «El Tesoro de Covarrubias», Estudios de lexicografía española, Madrid, Paraninfo, 1987, pp. 97-110. Dolores Azorín Fernández, «Datos para la historia de la lexicografía española. A propósito de las ampliaciones y desartollos del Tesoro de Sebastián de Covarrubias», Analecta Malacitana, XI-1, 1988, pp.117-124.

17 Recordemos que la Academia Española interrumpió la publicación de esta obra en 1770 para centrar sus esfuerzos en la confección del llamado «Diccionario vulgar» - hoy DRAE (Diccionario de la Real Academia Española)-, cuya primera edición aparecería en 1780. Cfr. Manuel Alvar López, «El caminar del diccionario académico», Actas del IV Congreso Internacional de EURALEX, Barcelona, Biblograf, 1992, pp. 3.27.

18 M. Seco, «Prólogo» en Estudios de lexicografía española, ed. cit., p.9.

19 Vid. nota 4. 
La falta de continuidad que había venido caracterizando a los diccionarios del español, se quiebra precisamente en el periodo que nos ocupa como lo demuestran la profusión de autores y de obras que salen a la luz a partir del segundo cuarto del XIX. El hilo conductor de este movimiento lexicográfico aunque resulte paradójico- será el Diccionario «vulgar» de la Academia, esto es, el DRAE que, desde su publicación en 1780 y con varias ediciones ya en su haber $^{20}$, constituía - a pesar de sus carencias- el primer proyecto lexicográfico sólido y continuado de la historia de la filología castellana ${ }^{21}$.

Como hemos señalado anteriormente, la mayoría de los autores que integran la corriente extra-académica se basarán en la edición del DRAE vigente en cada momento. Así, por ejemplo, Núñez de Taboada adiciona la sexta (1822), Peñalver la octava (1837), Salvá la novena (1843), etc., coincidiendo todos en destacar «como defecto mayor del Diccionario académico la cortedad de su repertorio» ${ }^{22}$, hecho que por sí solo justica la oportunidad de sus obras que, consecuentemente, saldrán a la luz con sensibles incrementos de voces y acepciones ${ }^{23}$.

No hay que olvidar, finalmente, que el origen de este movimiento lexicográfico estuvo presidido por intereses marcadamente comerciales, lo que vendría a explicar, en parte, el oportunismo y la escasa calidad de algunos de sus productos. Así, para M. Seco:

Los primeros pasos en esta dirección se habían dado cuando (...) los editores franceses descubrieron las posibilidades que les brindaba el mercado de las recién emancipadas colonias españolas de América -roto el comercio de ellas con España-, así como la inestimable colaboración de la propia metrópoli al suministrarles suficiente número de intelectuales exiliados útiles

20 Recordemos que, antes de rebasar el primer cuarto del siglo XIX, la Academia había sacado a la luz seis ediciones de su Diccionario, publicadas en $1780,1783,1791,1803,1817$ y 1822. Quedaba patente, pues, el empeño de la docta corporación por consolidar su obra, dotándola de la necesaria continuidad.

21 Para un seguimiento detallado de la evolución del Diccionario académico, remitimos a la obra de M. Alvar López, «El caminar del diccionario académico», ed. cit. Sobre el mismo tema véase también : M. Alvar Ezquerra, «El diccionario de la Academia en sus prólogos», La lexicografía descriptiva, Barcelona, Biblograf, 1993, pp. 215-239.

22 M. Seco, «El nacimiento de la lexicografía moderna no académica», ed. cit., p. 133.

23 La aportación real que estos autores realizaron en sus respectivas obras es difícil de precisar. Hasta el momento sólo contamos con muestreos parciales practicados en algunas de ellas (Cfr. a este propósito R. Baquero, «Notas en contribución a la historia de la lexicografía española monolingüe del siglo XIX», ed. cit.) y, por supuesto, con las declaraçiones que los mismos lexicógrafos efectúan en las portadas y prólogos de sus diccionarios y que, naturalmente, hay que manejar con mucha precaución pues su objetivo, la mayoría de las veces, no es informar al usuario sino poner de relieve su superioridad frente al Diccionario de la Academia (M. Seco proporciona cumplida noticia sobre este particular en «El nacimiento de la lexicografía modema no académica», ya citado). 
para desempeñar la necesaria tarea redactora. ${ }^{24}$

De todos modos, esta circunstancia -implícita, por otra parte, en la producción de diccionarios ${ }^{25}$ - no llegó a impedir que de este conjunto surgieran obras de indudable mérito. Pero, lo más importante del caso fue la instauración, por primera vez en España, de un incipiente pluralismo en la producción de diccionarios que, finalmente, traería como consecuencia el ensayo de nuevas formas de concebir el diccionario de la lengua ${ }^{26}$.

\section{La producción lexicográfica de Vicente Salvá}

Vicente Salvá es, sin duda, el mejor exponente del periodo que examinamos. En él concurren todas las circunstancias que hicieron posible el surgimiento de la corriente lexicográfica no académica del XIX: liberal exiliado, intelectual de reconocido prestigio, profundo conocedor de nuestra lengua y, por tradición familiar, de los entresijos del mercado editorial. Comenzó su andadura como lexicógrafo publicando, en 1832, el Diccionario latino-español de Valbuena ${ }^{27}$, pero pronto se sumaría al movimiento iniciado por los editores franceses, publicando una versión corregida por él mismo de la octava edición (1837) del Diccionario de la Academia. Se inicia así su contribución a la lexicografía monolingüe castellana,cuyos resultados pasamos a detallar a continuación:

1.-Diccionario de la lengua castellana, por la Academia española, reimpreso de la octava edición publicada en Madrid en 1837, con algunas mejoras por D. Vicente Salvá, París, J. Smith, 1838.

2.-La misma obra, segunda edición, mucho más correcta que la primera, según se demuestra a continuación de la Advertencia preliminar, París, J. Smith, 1841.

24 M. Seco, «El nacimiento de la lexicografía moderna no académica», cit., p. 130.

25 No hay que olvidar que el diccionario, aparte de su finalidad didáctica, es también un producto comercial y como tal sujeto a los avatares que dicta el mercado en cada momento. Para Alvar Ezquerra, «..otra de sus características primordiales (es) la de ser un producto manufacturado. La industria del libro lo considera de esta manera, y lo comercializa como si se tratase de cualquier otro objeto». ( $L$ La lexicografía en los últimos veinte años», La lexicografia descriptiva, Barcelona, Biblograf, 1993, p. 14).

26 Véase lo que apuntábamos sobre este aspecto en : D. Azorín Fernández, «La lexicografía española en el siglo XIX. Desarrollos y tendencias», Actas del I Congreso de Lingüistica General, Valencia, 15 al 17 de febrero de 1994, (en prensa).

27 Diccionario latino-español, dispuesto por D. Manuel de Valbuena. Séptima edición, corregida y considerablemente aumentada por Don Vicente Salvá, París, J. Smith, 1832. De esta obra, que conoció numerosísimas ediciones posteriores, Salvá realizaría cinco ediciones más en 1834, 1837, 1840,1843 y 1846 . 
3.-Nuevo diccionario de la lengua castellana que comprende la última edición íntegra, muy rectificada y mejorada, del publicado por la Academia española, y unas veinte y seis mil voces, acepciones, frases y locuciones, entre ellas muchas americanas, añadidas por D. Vicente Salvá, París, H. Foumier y Cía, $1846 .^{28}$

4.-La misma obra, segunda edición, corregida y mejorada, París, H. Fournier y Cía, $1847 .^{29}$

5.-La misma obra, tercera edición, París, Garnier, $1852 .{ }^{30}$

6.-La misma obra, cuarta edición, París, Garnier, $1854 .^{31}$

7.-La misma obra, París, Garnier, $1857 .{ }^{32}$

8.-La misma obra, séptima edición, añadida con un suplemento que contiene la voces de ciencias, artes, etc., París, $1865 .^{33}$

9.-La misma obra, octava edición, París, Garnier, $1879 .{ }^{34}$

La restante información que hemos podido allegar acerca de las ediciones del Nuevo Diccionario de Salvá es sólo indirecta. Así, no hemos constatado la existencia de la edición de Méjico, Blanquel, 1953 que Palau da como probable. Tampoco la de México, Blouquel, 1881 que menciona Fabbri ${ }^{35}$.

Las numerosas ediciones de que fue objeto esta obra son muestra evidente de la notable difusión de la misma, seguramente más en los países de la América latina que en España. No en balde Salvá destinaría su Diccionario a los hispanohablantes de nuestras ex-colonias:

28 Es un grueso volumen en octavo mayor. El único ejemplar impreso en amarillo acartulinado que lleva un retrato de Salvá, se encuentra en la Biblioteca Municipal de Valencia (fondo Carola Reig 13). Se puede consultar esta misma obra, en cuarto en la Bibliothèque National de París (X.5293), así como en la British Library (12941.p.2).

29 También en octavo mayor. Hay un ejemplar en la Biblioteca Municipal de Valencia (Fondo Carola Reig 354). Hemos localizado otro ejemplar en la Biblioteca Nacional de Madrid (1/40045) y otro más en la Bibliothèque National, en cuarto, (X.5294).

30 En cuarto. Empleamos como fuente el Catalogue Général de la Bibliothèque National (X.5295).

31 En cuarto. Bibliothèque Nationale (X.5296).

32 Nuestra fuente es el fichero de la Biblioteca Nacional (1/40045).

33 Noticia extraída de The British Library General Catalogue, donde figura con la signatura 12943.g.1. Yerra Palau al asignar la autoría del «Suplemento» a Vicente Salvá, muerto como estaba desde hacía varios años (1849).

34 En cuarto. Ejemplar localizado en la Bibliothèque Nationale (X.1276).

35 Esta última edición es de dudosa inclusión por su número de páginas -760 , según Fabbri-, teniendo en cuenta que la obra original de Salvá 1140 páginas, más el Suplemento posteriormente añadido de al menos 327. Cfr. M. Fabbri, A Bibliography of Hispanic Dictionaries, Imola, 1979, p.70. 
Entre las dicciones olvidadas por la Academia en su diccionario -escribe Salvá-, debieron llamar mi particular atención desde que me propuse adicionarlo las peculiares de América, porque se hacía más notable la sinrazón de excluirlas en una obra destinada principalmente a ella. Y aún podía tachárseme de ingrato, si no aprovechaba esta coyuntura de corresponder a la singular preferencia con que ha acogido cuantos libros he publicado. ${ }^{36}$

\section{EI «Nuevo diccionario de la lengua castellana».}

Podemos considerar al Nuevo diccionario como la obra de madurez de Vicente Salvá ${ }^{37}$. Como afirma Alvar Ezquerra «en él queda reflejado el interés que sentía por el léxico (...) y las múltiples anotaciones que había hecho a lo largo de cuarenta y seis años ${ }^{38}$. Publicado sólo tres años antes de su fallecimiento, nuestro autor reune aquí el bagaje de toda una vida dedicada al estudio de la lengua y la literatura españolas. Valgan sus propias palabras para dar cuenta de la lenta y meditada labor de la que fue fruto su Diccionario:

Los estudios de toda mi vida, la lectura (...) y los varios trabajos que han visto la luz pública (...) me han obligado a recurrir de continuo al Diccionario de la Academia, para consultar las dificultades que se me ofrecian, han debido producir un sinnúmero de anotaciones acerca de los descuidos $u$ olvidos que de paso observaba. Estuvieron diseminadas en varios papeles sueltos, hasta que en 1833 entreví la posibilidad de poderlas publicar, $y$ entonces las junté en un volumen (...) Por manera que la colección actual de mis apuntes abraza un periodo de cuarenta y seis años ${ }^{39}$.

Salvá no realiza un diccionario de nueva planta. Corrige y adiciona la novena edición del DRAE (1843), «..pero es tal el número de modificaciones introducidas (...) que podemos considerarlo una obra original $»^{40}$. Así, aunque su primera intención fue componer un suplemento con todas las informaciones que había ido reuniendo, añadiéndolo como apéndice a su edición del DRAE, pronto

36 Vicente Salvá, «Introducción del adicionadon», Nuevo diccionario de la lengua castellana, París, 1846, p.XXVII.

37 El propio Salvá, en la «Introducción del adicionador» que precede a su Nuevo diccionario, escribía lo siguiente: «Siempre he creído que el hombre debe estudiar y meditar mucho en los dos primeros tercios de su vida, para poder en el último producir algo que por su sencillez, buen método o mayor cúmulo de noticias, merezca preferirse a lo que otros han publicado» (Salvá, "Introducción», p. VII).

38 V.Salvá, «Introducción del adicionadon», Nuevo diccionario.. ed. cit., p. VII. Las referencias a este texto se harán en adelante en el cuerpo principal del artículo, seguidas de la leyenda "Salvá, Introducción, p. ..

39 M. Alvar Ezquerra, «Tradición en los diccionarios del español», ed. cit., p. 20.

40 Cfr. a este respecto, los argumentos del propio Salvá en su «Introducción..», p. XXI. 
descartaría esta manera de presentación de sus propios materiales por considerarla incómoda en extremo para sus lectores ${ }^{41}$. De manera que, finalmente, se decidió a incorporar su contribución original al cuerpo del texto académico, pero convenientemente deslindada de éste a través de un sencillo sistema de marcas. Él mismo explica el procedimiento que siguió de la siguiente manera:

(...) la nona edición es más completa de vocablos y más purgada de defectos de redacción que ninguna de las anteriores, y yo no podía vacilar en adoptarla por texto e incorporar en ella mis adiciones, ya que no tengo espacio para construir un diccionario de planta. Me he decidido pues a reproducirla sin omitir cosa alguna, aumentándola con las voces, acepciones y frases, etc. que tenía recogidas, y rectificando las definiciones y correspondencias latinas; pero de modo que siempre aparezca lo que es de mi cosecha (...) Con este fin he deslindado mi trabajo del académico del modo siguiente: son enteramente míos los artículos que llevan la +; en los notados con un $*$ me pertenece todo lo que va inclus@ dentro de paréntesis cuadrados, y el / denota que se ha mudado el artículo del sitio que equivocadamente ocupaba, o se ha dado nuevo orden a sus partes, o se ha rectificado algo la redacción. (Introducción, p. XXI).

De la extensa cita que acabamos de reproducir se desprende que la labor de Salvá va más allá de la mera adición de materiales léxicos. Nuestro lexicógrafo incorporó artículos nuevos, sumó nuevas acepciones a los que traía el texto académico, corrigió definiciones, añadió la correspondiente marca de sanción a las acepciones que lo requerían, reorganizó la disposición de los artículos y, finalmente, corrigió las erratas tipográficas, sumando a la fe de erratas que la Academia consignó en su edición, otras muchas que se le pasaron por alto a la docta corporación y que Salvá había detectado tras su minucioso examen del Diccionario académico ${ }^{42}$.

Aunque el hecho de tener que trabajar sobre lo ajeno representaba una dificultad añadida a la tarea, ya de por sí costosa, de confeccionar un diccionario, Salvá consiguió plasmar, en su propia obra, muchos de los presupuestos teóricos que sustentaban su modelo de «diccionario ideal». Su doctrina lexicográfica quedó compendiada en la extensa «Introducción» —citada ya varias vecesque precede al Nuevo diccionario donde nuestro autor, haciendo gala de un

41 Cfr. las observaciones que hace Salvá sobre este aspecto en su «Introducción...», p. XXI.

42. A este respecto, recomienda concisión y claridad en las definiciones, sin alargarlas con "pormenores innecesarios» cuya «superfluidad confunde al lector» (Introducción, p.X). Aunque también es consciente de que «..dar una definición bastante exacta, en términos inteligibles y que no huelan a la pedantería de los que siempre hablan facultativamente, no es negocio tan fácil como lo cree el que nunca se ha parado a meditarlo. Cuanto más triviales y comunes son las cosas, más se toca la dificultad de definirlas. Todos saben, por ejemplo, qué es abrir, cerrar, dar cuerda a un reloj (...) Con todo pruebe cualquiera a decir en pocas palabras y de un modo acomodado a la inteligencia común de los lectores, lo que es cada una de dichas operaciones (...) y se hallará un poco embarazado» (Introducción, p. XXXII). 
rigor metodológico inusual en su época, expuso sus ideas acerca de los contenidos y de los aspectos formales de su modelo de diccionario de la lengua.

En lo que atañe a la aplicación de la técnica lexicográfica, merece la pena destacar sus puntualizaciones acerca de la calidad formal de las definiciones ${ }^{43}$, el orden de prelación de las mismas en el articulado del diccionario ${ }^{44} \mathrm{o}$ la intromisión de elementos ideológicos ajenos al contenido del definido. Sobre este último aspecto, Salvá se muestra taxativo al afirmar:

(...) un lexicógrafo nunca debe manifestar sus propensiones ni su modo de pensar en materias políticas y religiosas, ni menos ridiculizar o condenar como errores las doctrinas que siguen varones muy doctos (...) Este es el mejor medio para que sea leído por un largo periodo y por personas de todos los países y de diversas opiniones, y el más seguro para no equivocarse. (Introducción, p. XIV).

Del minucioso programa de revisión que Salvá llevó a cabo en el texto académico, destacan, sobre todo, las nuevas incorporaciones de materiales léxicos. Como reza en la portada de su obra, nuestro autor declara haber añadido «unas veinte y seis mil voces, acepciones, frases y locuciones», sobre las ya contenidas en el DRAE de 1843. Su proceder entronca aquí con lo que fue tónica general entre los lexicógrafos de su generación, es decir, superar el caudal de voces del Diccionario de la Academia.

La singularidad del Nuevo diccionario, frente a otros productos de su época, se muestra también en los criterios de selección del léxico que Salvá utiliza. Convencido de que el diccionario ha de ser un instrumento eficaz no sólo para enfrentarse a las novedades del presente, sino para acceder también a las dificultades que plantean nuestros clásicos, 0 a las que se derivan de las

43 Sobre este punto, critica el decuido de la Academia al no «guardar un mismo método para colocar las acepciones, pues infinitos nombres y verbos principian por la metafórica, otros por la provincial, estos por la anticuada, aquellos por la germanesca o facultativa..» (Introducción, p. XIV). En la nota 11 de esta misma página, señala Salvá el procedimiento que él ha seguido en la ordenación de las acepciones en los artículos de nuevo cuño que añade al DRAE: «He procurado colocar al fin las acepciones facultativas y provinciales, y después de ellas las anticuadas, menos cuando la técnica, desusada, etc. es la primera y fundamental de la voz, como en círculo, ladino, punto y zozobra, porque entonces claro es que debe principiar el artículo».

${ }_{44}$ El criterio restrictivo que mantuvo la Academia respecto de los neologismos de extracción foránea lo podemos detectar en el mismo «Prólogo» de su novena edición, la que Salvá utiliza como base. Las manifestaciones que, a continuación, reproducimos se inscriben en la línea del casticismo idiomático que suscribe la corporación: «Este es el objeto primordial del Diccionario, dar a conocer las palabras propias y adoptivas de la lengua castellana, sancionadas por el uso de los buenos escritores; pero muchos no lo entienden así; y cuando no encuentran en el Diccionario una voz que les es desconocida, en vez de inferir que no es legítima y de buena ley, lo que infieren es que el Diccionario está diminuto. Así hemos visto lamentarse algunos de no hallar comité por comisión, secundar por cooperar, y otras muchas extranjeras, de que están infestados la mayor parte de los escritos que diariamente circulan, y que todo el mundo lee por la importancia de los asuntos sobre que versan» («Prólogo» de la Real Academia Española, a su Diccionario de la lengua castellana, Madrid, 1843, tomado del Nuevo diccionario... de V. Salvá, ed. cit., p. V). 
diferencias dialectales en el uso de la lengua, nuestro autor plantea un programa de incorporaciones léxicas capaz de satisfacer las expectativas de una variada comunidad de usuarios. Esta amalgama de características sitúan al Nuevo diccionario en el cruce de dos tradiciones aparentemente contrapuestas: la histórica y la descriptiva.

Con el fin de mostrar, sobre datos fiables, la absoluta coherencia que Salvá mantiene en la práctica respecto de sus declaraciones programáticas, hemos delimitado un corpus, a modo de muestra representativa, constituido por todas las voces originales de nuestro autor incluidas en la letra A de su Nuevo diccionario.

Los datos obtenidos en la muestra, se distribuyen de la siguiente manera:

NÚMERO TOTAL DE VOCES......................1342

VOCES NO MARCADAS.....................383

VOCES MARCADAS.......................941

DIACRÓNICAMENTE.................658

Antiguas....................609

Poco uso..................... 27

Neologismos................ 22

DIATÓPICAMENTE................. 72

Americanas.................. 55

Peninsulares................ 17

DIAFÁSICAMENTE................. 18

Familiares................ 18

DESPLAZAMIENTOS SEMÁNTICOS....... 54

Metafóricas................. 7

Jocosas...................... 14

De capricho.................. 33

DIATÉCNICAMENTE.

Las cifras anteriores confirman, en efecto, la absoluta fidelidad entre el programa de revisiones que Salvá traza en la Introducción y su puesta en práctica en el cuerpo de la obra. Así, si uno de los principales defectos que destaca del DRAE es su incapacidad para dar satisfacción a quienes lo consultan con el fin de auxiliarse en la lectura e interpretación de nuestros autores clásicos, no es de extrañar que el grueso de sus adiciones se encamine al acopio de voces antiguas que, como se aprecia en nuestra muestra, es el subconjunto de voces marcadas con mayor representación. Queda justificada, con creces, la advertencia de la Introducción que seguidamente reproducimos: 
Con el diccionario de la Academia en la mano apenas podría darse un paso, no diré en la lectura del Fuero Juzgo, de los poetas anteriores al siglo $\mathrm{XV}$, o de las obras de lenguaje de abstruso, como la Picara Justina o alguna de Quevedo; sino de las que o manejan todos por gusto o son las primeras en ciertas facultades, pues ni el jurisconsulto podría entender las partidas (...) ni algunos pasajes del Novísima; ni el médico estudiar los problemas de Villalobos, ni el aficionado a las bellas letras las Coplas de Mingo Revulgo, al príncipe de los poetas de su siglo, Juan de Mena, ni, lo que parecerá más increíble, los escritos de autores tan castizos y de época muy posterior como lo son Alemán y Cervantes. (Introducción, p.XXV).

Al suplir esta carencia, cumple Salvá con uno de sus principales objetivos, enunciado ya al comienzo de su Introducción: «...porque para entenderlos - se refiere a nuestros clásicos-es para lo que consultan por lo común el diccionario los españoles; y más particularmente los extranjeros» (p.XI).

La misma finalidad - leer a los clásicos-desempeñan las voces metafóricas y, particularmente, las que él bautiza como «de capricho»o las del estilo jocoso.

A pesar de esta decantación culturalista que convierte al diccionario en instrumento para acceder a nuestra tradición literaria, las restantes incorporaciones léxicas de Salvá vienen a ser la prueba palpable de la vertiente sincrónicodescriptiva presente también en su obra. Las voces del estilo familiar y, sobre todo, los neologismos y los vocablos de extracción técnica se inscriben en esa dirección que denota, en nuestro autor, una mayor flexibilidad a la hora de registrar los usos comúnmente extendidos, frente al purismo que caracteriza a la Academia ${ }^{45}$.Especialmente en el capítulo de las voces téc icas, la crítica que realiza Salvá al duro criterio restrictivo de la corporación, se inserta en una línea de denuncia más amplia del inmovilismo académico que permite situaciones como la descrita por nuestro autor en el párrafo siguiente:

¿Cómo puede explicarse (...) que la Academia (...) nos dé como corrientes millares de voces anticuadas, al paso que deja de admitir las que todo el mundo conoce y usa? ¿Qué otra razón puede asignarse para que el Diccionario no se halle, en punto a ciencias y arte, no diré al nivel de los conocimientos de los Académicos, lo cual sería sobrado pedir, sino a la par de los progresos comunes y generalizados? El que registre su última edición, creerá que en España no se tenía noticia en 1843 del alumbrado de gas, de los reverberos, de las prensas hidráulicas, de los ferrocarriles, de los puentes suspendidos, ni de los barcos de vapor..» (Introducción, p.VIII).

Resta por mencionar, finalmente, una de las características más novedosas del Nuevo diccionario: nos referimos a la incorporación masiva de voces provinciales del español de América. Las conclusiones provisionales que revela la muestra, - donde éstos adquieren una espectacular representación frente a los dialectalismos peninsulares-, podemos elevarlas a definitivas tras el estudio pormenorizado que realizamos de este subconjunto léxico en la totalidad del 
Nuevo diccionario ${ }^{45}$. Según nuestros cómputos, Salvá incorporó a su Diccionario un total de 1543 americanismos, entre voces y acepciones, superando, con mucho, las aportaciones que, en este terreno, realizaron sus predecesore ${ }^{46}$. Este abultado número de voces americanas demuestra cómo el propósito de Salvá, expresado varias veces en su Introducción ${ }^{47}$, de dar carta de naturaleza a los provincialismos de América, no quedó en mera declaración de intenciones, propiciada por su deseo de complacer a sus lectores del otro lado del Atlántico.

A la vista de los rasgos que hemos ido exponiendo, podemos concluir que el Nuevo diccionario de la lengua castellana es el primer intento de diccionario total o extensivo de la historia de nuestra lexicografía. Su amplitud de criterios en la selección del léxico — más orientada hacia el uso que a lo normativo- 10 sitúan en la órbita de los diccionarios descriptivos. Aunque su característica privativa - la gran atención prestada a las voces antiguas - hace de él un producto híbrido, encrucijada entre dos tradiciones lexicográficas: la histórica y la sincrónico-descriptiva. Por último, el riguroso método empleado en su confección y la objetividad manifiesta en el tratamiento de la información lo convierten en el primer ejemplo de diccionario moderno de la historia de la lexicografía española monolingüe.

45 Cfr. D. Azorín y R. Baquero, «Los americanismos en el Nuevo diccionario de la lengua castellana de Vicente Salvá», ed. cit.

46 Poco más de un centenar de americanismos incluyeron nuestros primeros académicos en el Diccionario de Autoridades (Cfr. Aurora Salvador Rosa, "Las localizaciones geográficas en el Diccionario de Autoridades», Lingüística Española Actual, 7, 1985, pp.103-139). Casi doscientas hemos contabilizado en el Diccionario de Terreros y Pando.

47 Vid. «Introducción», p. XIV, XXVII y XXVIII. 